\title{
Utilización de Lessons como herramienta de apoyo a la docencia inversa en la asignatura de Biofísica
}

José A. Gómez-Tejedor ${ }^{\mathrm{a}}$, José Molina Mateob ${ }^{\mathrm{b}}$, María-Antonia Serrano ${ }^{\mathfrak{c}}$, José M. Meseguer-Dueñas $^{d}$, Ana Vidaurre ${ }^{e}$ y Jaime Riera ${ }^{f}$

${ }^{a}$ Departamento de Física Aplicada. ETS de Ingeniería del Diseño. Universitat Politècnica de València. Camino de Vera, s/n. 46022 Valencia, Spain, jogomez@fis.upv.es, bjmmateo@fis.upv.es ํmserranj@,fis.upv.es,

\begin{abstract}
The use of the PoliformaT tool has been analysed, both in terms of the number of visits and their temporary distribution. As an example, the temporary pattern of visits to some videos has also been analysed. Both patterns have been analysed according to the date of the examination on the topic. In addition, an opinion survey has been carried out in which the students have been asked about the methodology and the tools and means available. The results indicate that the students work regularly with a good organization of time. They visit the page continuously with a slight increase the days before the exam, while they visit the videos earlier, more linked to the presentation of the corresponding topic.
\end{abstract}

Keywords: Learning platforms, Lessons, videos, flip teaching.

\begin{abstract}
Resumen
Se ha analizado el uso de la herramienta de PoliformaT, tanto el número de visitas como su distribución temporal. También se ha analizado, a modo de ejemplo, el patrón temporal de visitas a algunos vídeos. Ambos patrones se han analizado en función de la fecha del examen sobre esa materia. Además se ha realizado una encuesta de opinión en la que los estudiantes han valorado la metodología y las herramientas y medios disponibles. Los resultados indican que los alumnos trabajan de forma regular con una buena organización del tiempo. Visitan la página de forma continua con un ligero incremento los días antes del examen, mientras que visitan los vídeos con mayor antelación, más ligado a la presentación del tema correspondiente.
\end{abstract}

Palabras clave: Plataformas de aprendizaje, Lessons, vídeos, docencia inversa. 


\section{Introducción}

La Docencia Inversa (DI) se ha vuelto cada vez más popular en la educación superior desde que se describió por primera vez como un "aula invertida" en el año 2000 (Lage, 2000). Esta metodología cambia completamente la estructura y organización de la docencia: los alumnos preparan la sesión de clase previamente mediante la lectura de documentación o visualizando videos, de manera que el tiempo de la clase se dedica al trabajo y discusión en equipo y a la realización de tareas dirigidas por el profesor (Garrison, 2013). La DI se basa en modelos de aprendizaje activo (Bonwell 2005; Meyers 1993) y es un término muy general que cubre una amplia gama de actividades y estrategias de aprendizaje y modelos docentes.

En la Universitat Politècnica de València, la Docencia Inversa está teniendo un impulso institucional muy importante, de tal manera que dispone de un blog desarrollado por expertos en la materia (https://docenciainversa.blogs.upv.es/el-proyecto/proyecto-clase-inversa-upv/) y ha sido noticia destacada dentro de la web de la UPV (http://www.upv.es/noticiasupv/noticia-6615-clase-inversa-o-es.html). Incluso, en el sobre virtual de matrícula de algunos centros de la UPV que los futuros estudiantes consultan en el momento de su matriculación disponen de información relativa a esta metodología: véase, por ejemplo, el sobre virtual de matrícula para los másteres de "Arquitectura Avanzada, Paisaje, Urbanismo y Diseño", “Arquitectura del Paisaje” y "Conservación del Patrimonio Arquitectónico" de la ETS de Arquitectura (http://www.upv.es/contenidos/SMAT/etsa/1041403normalc.html).

Este proyecto de Docencia Inversa en la UPV comenzó en el curso 2014-15 con un grupo piloto en el grado de Administración y Dirección de Empresas y otro en el Grado en Ingeniería Informática (http://www.upv.es/noticias-upv/noticia-6615-clase-inversa-oes.html) habiendo tenido desde entonces resultados muy positivos, tanto desde el punto de vista de los alumnos, reflejado en encuestas de opinión y mejora de los resultados, como desde el punto de vista de los profesores involucrados (Argente, 2016; Terrasa, 2015; GómezTejedor, 2019) y en concordancia con otros estudios previos realizados en otras universidades (Lemley, 2013; Valverde, 2014).

Se han desarrollado muchos tipos diferentes de modelos de DI, cada uno con una perspectiva u orientación diferente. En los últimos años, un eje conductor importante en estos modelos de DI, sobre todo en el ámbito de la educación superior, ha sido la utilización de plataformas de aprendizaje basadas en las Tecnologías de la Información y la Comunicación (TIC). Los efectos de la aplicación de la DI ha sido analizada en numerosos trabajos; entre ellos: (Chen, 2014; Jovanovic, 2019; Tomas, 2019; Vo, 1017; Sun 2018)

De esta forma, las plataformas de gestión de aprendizaje basadas en TICs ofrecen una amplia gama de herramientas y desempeñan un papel importante en el diseño de la DI. Las universidades han adoptado diferentes plataformas diseñadas específicamente para fines educativos, como por ejemplo Moodle y Sakai. En concreto, la Universitat Politècnica de València (UPV) ha optado por la plataforma PoliformaT (https://poliformat.upv.es/portal) adaptada de Sakai (https://sakaiproject.org/). La DI aprovecha el hecho de que las plataformas de aprendizaje pueden organizar el contenido del curso en unidades temáticas, videos, tareas, evaluación, etc. (Ardid, 2015).

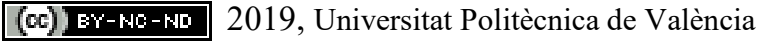


En particular, en este trabajo se presenta la utilización de la herramienta Lessons de PoliformaT para la organización del material docente de un curso de la asignatura de Biofísica de la ETS de Ingenieros Industriales de la UPV, que se imparte mediante la metodología de Docencia Inversa. Se ha utilizado las posibilidades de análisis de accesos que dispone la herramienta Lessons para estudiar la evolución en el tiempo del acceso de los alumnos a la plataforma y a los diferentes contenidos en la misma, y la relación de estos accesos con fechas clave de la asignatura como son días de clase, entrega de trabajos o exámenes. Además se ha pasado una encuesta de opinión sobre temas relacionados con la docencia inversa y las herramientas utilizadas.

\section{Objetivos}

- Valoración de la utilidad de la herramienta Lessons para organizar la docencia inversa.

- Estudio de la distribución temporal del acceso a la plataforma de aprendizaje y al visionado de vídeos.

- Análisis de la opinión de los estudiantes en relación con la docencia inversa y las herramientas utilizadas.

\section{Desarrollo de la innovación}

Se trata de una asignatura obligatoria, de segundo curso, de la titulación de Ingeniería Biomédica. En este caso, la asignatura se ha organizado por unidades temáticas, dentro de Lessons, y en cada unidad temática se organiza el material en forma de documentos, vídeos y ejercicios de autoevaluación. Éstos últimos en formato de Exámenes de PoliformaT.

La metodología en clase sigue el modelo de DI. Se propone a los estudiantes que visualicen los vídeos (a veces antes de la sesión de clase, a veces con posterioridad), que lean determinados documentos o que realicen exámenes de autoevaluación. En las clases se realizan actividades que complementan el trabajo que los estudiantes han realizado previamente en casa. Se discuten los conceptos (más que explicarlos con todo detalle); se aclaran las dudas que les han surgido al realizar los ejercicios de autoevaluación; y se plantea el realizar actividades de profundización que realizan en grupos. En genral, se trata de alumnos muy motivados que participan activamente en las actividades que se les preoponen. En las prácticas de laboratorio se propone la misma estrategia. Con anterioridad a la sesión, los alumnos tienen que preparar la práctica con el material disponible (vídeos y documentos) y tienen que completar un pequeño examen de evaluación que contribuye en un $10 \%$ a la calificación de la práctica.

En el modelo actual, aproximadamente el 50\% del tiempo de clase se dedica a desarrollar contenidos, y el $50 \%$ a resolver problemas prácticos que surgen del trabajo en casa de los estudiantes.

Para validar el modelo DI+Lessons, habría que verificar que los estudiantes trabajan de forma continua y autónoma. Esto se puede comprobar mediante la estadística de visitas a los diferentes recursos: visitas a lessons, visionado de vídeos, realización de los exámenes de 
autoevaluación, etc. Además se ha pasado una encuesta en la que los estudiantes valoran la utilidad de la herramienta y aportan información del modo en que la utilizan.

\section{Resultados}

Con objeto de analizar el funcionamiento de la herramienta Lessons en el contexto de DI, realizamos una doble tarea. En primer lugar, investigamos la utilización que se ha hecho de esta herramienta por parte de los alumnos. Para ello nos basamos en el recurso Estadística, propia de la plataforma PoliformaT que se utiliza en la UPV. Mediante la misma, podemos acceder al número de visitas de los alumnos a los distintos recursos de la plataforma. Por otro lado, analizamos una encuesta de satisfacción proporcionada a los alumnos, sobre la herramienta Lessons y el entorno de DI en el que se ha desarrollado su aprendizaje.

\subsection{Uso de la herramienta en el curso}

Como un índice de utilización de Lessons por los alumnos, tomamos el número de visitas a los distintos recursos a lo largo del curso, así como la distribución de esa utilización por alumno.

\subsubsection{Utilización de Lessons a lo largo del curso}

En la Fig.1 se muestra el promedio de visitas recibidas por alumno a lo largo de todo el curso, por hora de clase recibida. Las visitas por alumno durante el curso han sido 248 , lo que supone 6 visitas por alumno y hora lectiva. Sin embargo, Desde el comienzo del curso, en el mes de septiembre, las visitas van disminuyendo, desde 115 en promedio durante el primer mes a 7 en el último del curso, lo cual se puede explicar en parte por el hecho de que gran parte de los recursos visitados admiten su descarga.

Es muy significativo el hecho de que en el segundo mes la visitas caen a menos de la mitad de las recibidas en el primer mes. Esta circunstancia, ya observada en otros trabajos (Vidaurre, 2018), puede estar relacionada con el efecto "novedad" de su utilización, en su doble vertiente: falta de destreza en su utilización, así como alta motivación frente a lo nuevo. 


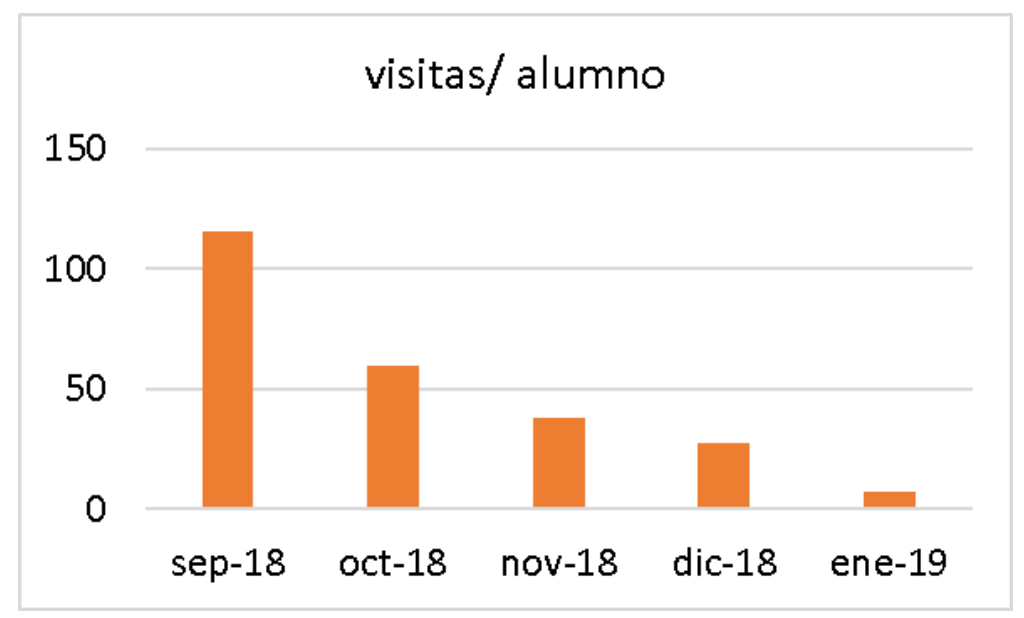

Fig. 1 Visitas/alumno a lo largo del curso

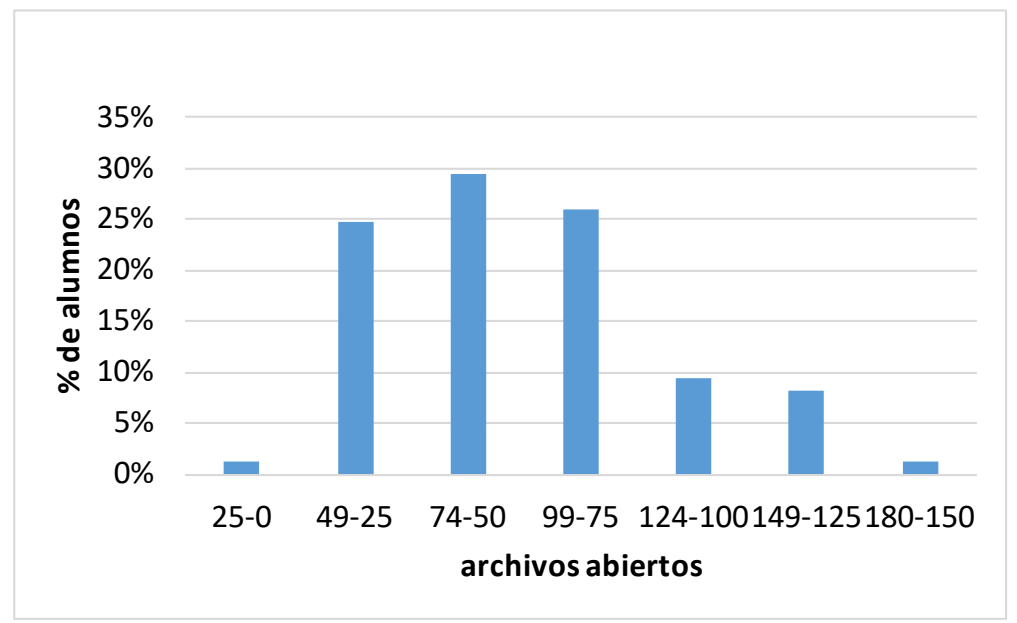

Fig. 2 Frecuencia de visitas por alumno

En la Fig. 2 se sintetiza la distribución de las visitas por alumno. Un análisis pormenorizado de la misma nos mostraría una amplia dispersión en el uso de la herramienta de unos alumnos a otros, lo cual no nos puede sorprender. Sin embargo, desde el punto de vista de análisis global del curso, nos puede interesar detectar la existencia o no de grupos que se autoexcluyen de la marcha del curso. Los resultados obtenidos muestran que más de la mitad de los alumnos abren entre 50 y 100 documentos y sólo un porcentaje muy bajo no alcanzan a los 25 documentos consultados. Estos datos confirman la participación generalizada de los estudiantes en la marcha de la asignatura, de la que se excluye un porcentaje muy bajo, que se corresponde con muy reducida tasa de abandonos $(2,4 \%)$.

\subsubsection{Patrones de uso de Lessons}


Para analizar los patrones de conducta de utilización de Lessons en las tareas cotidianas de la asignatura, se ha obtenido el promedio de visitas por día de la semana, a lo largo de todo el curso (véase Fig. 3). Los resultados obtenidos muestran una distribución del trabajo homogénea, a lo largo de la semana, sin que se marquen picos de trabajo en los días anteriores a las clases presenciales (tarde del miércoles y mañana del viernes). El patrón de trabajo sugiere una distribución semanal (más actividad previa a la primera clase semanal), en la línea de los objetivos planteados.

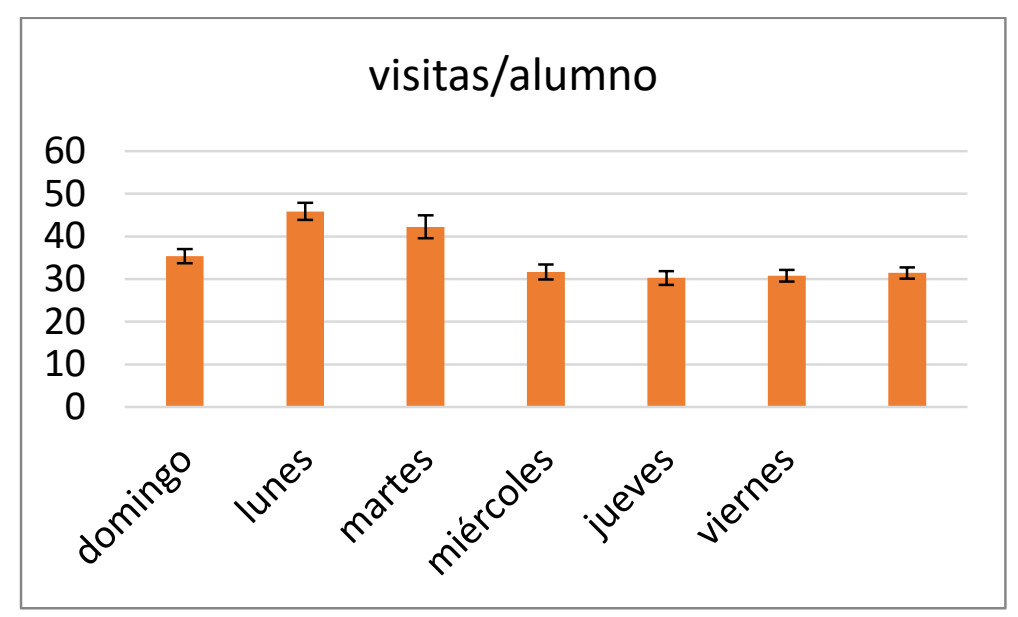

Fig. 3. Frecuencia de visitas por día de la semana

Complementariamente al patrón semanal de trabajo, es importante conocer la posible existencia de patrones de comportamiento relacionados con las cuatro pruebas evaluatorias que tienen lugar a lo largo del curso. Con esta finalidad, representamos el número de visitas en fechas próximas cada uno de los exámenes. En la Fig. 4 se constata que los accesos de los alumnos se incrementan en las fechas próximas a los exámenes. Al analizar las dos semanas previas a la realización del examen, el patrón parece el mismo: la frecuencia mantiene un ligero aumento sostenido durante el periodo estudiado, incrementándose algo más en los dos últimos días previos al examen. Las visitas previas al primer examen presentan, sin embargo, una característica particular, con un pico muy notable en el día" -2". La explicación a ello cabe encontrarla en el nerviosismo e inseguridad propio de una primera prueba. 


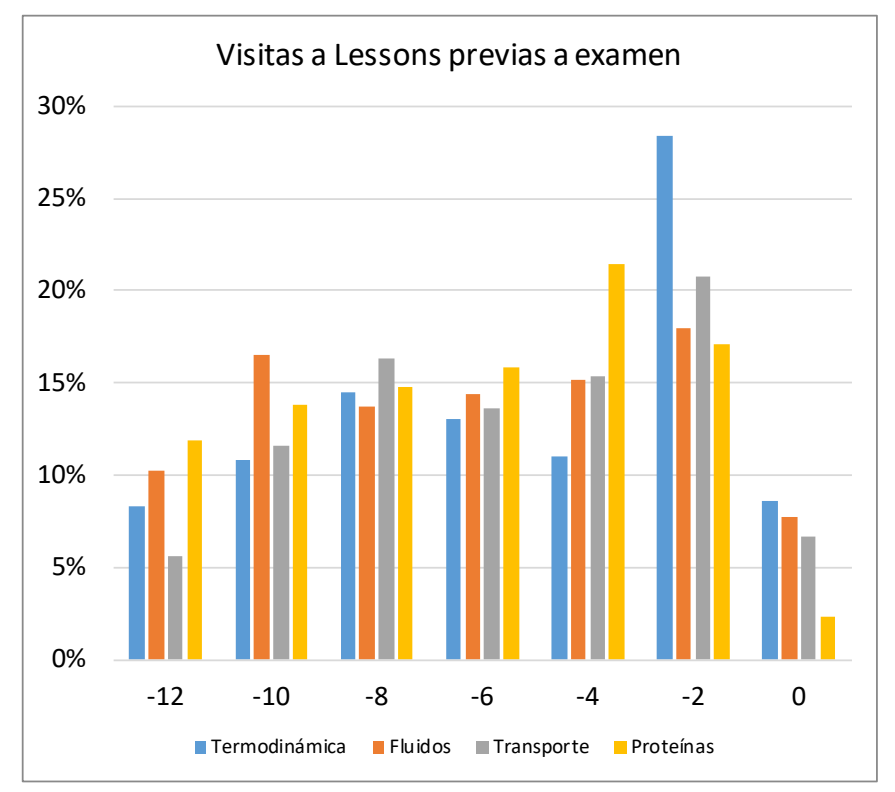

Fig. 4. Visitas previas a las distintas pruebas

En relación a la visita de los vídeos, podemos apreciar en la Fig. 5 que el patrón es diferente. Los vídeos se visualizan preferentemente alrededor de la fecha en la que se presenta el tema, presentando muy pocas visitas en los días previos al examen. Esto indica que los vídeos son utilizados como material de trabajo inicial en la introducción de un tema, mientras que a medida que trascurre el tiempo utilizan otros materiales como los exámenes de autoevaluación o los documentos.

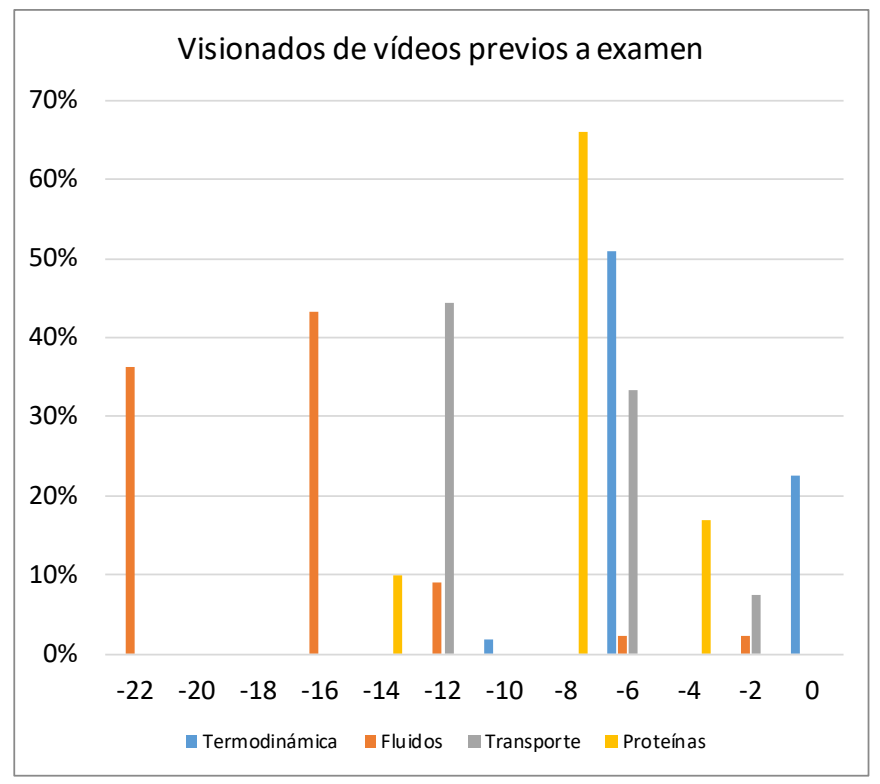

Fig. 5. Visitas a los vídeos previas al examen 


\subsection{Opinión de los alumnos}

Con objeto de conocer la opinión de los alumnos sobre el entorno de DI en el que se ha desarrollado su aprendizaje, se les ha pasado una encuesta, con las siguientes preguntas:

P1- Mi nivel actual de motivación por la materia es...

P2- La distribución de tiempo en las clases de teoría de aula me parece que debería de ser...

P3- Me sirve de ayuda para preparar la asignatura...

P4- El trabajo en equipo, realizado con mis compañeros, me ha sido de gran utilidad para...

P5- Se podría mejorar...

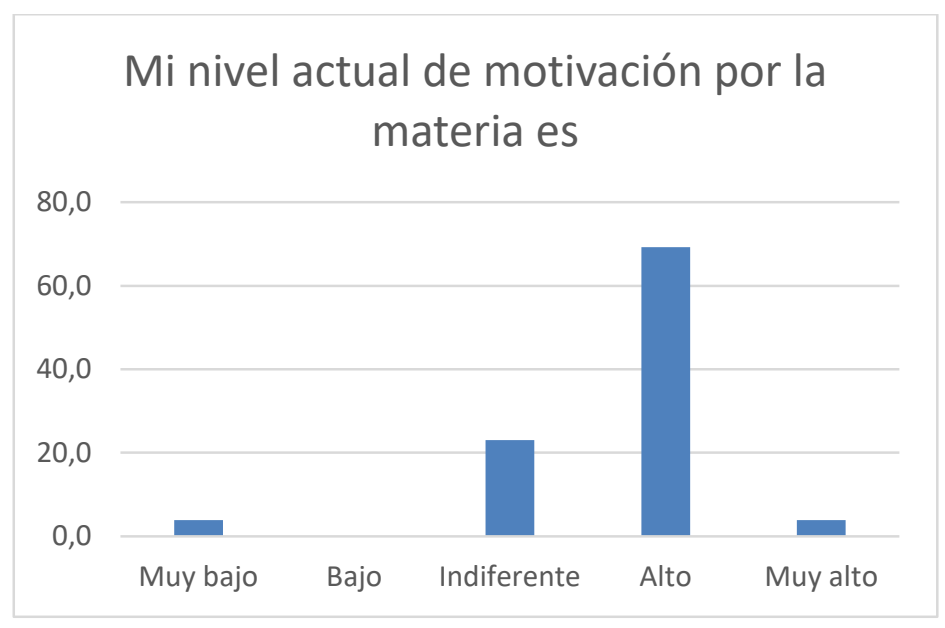

Fig. 6. P1:Mi nivel actual de motivación por la materia es...

El nivel de motivación por la asignatura es alto $(6,7 / 10)$, siendo pequeña la dispersión ( DS=1,8), tal como se aprecia en la Fig. 6 . 


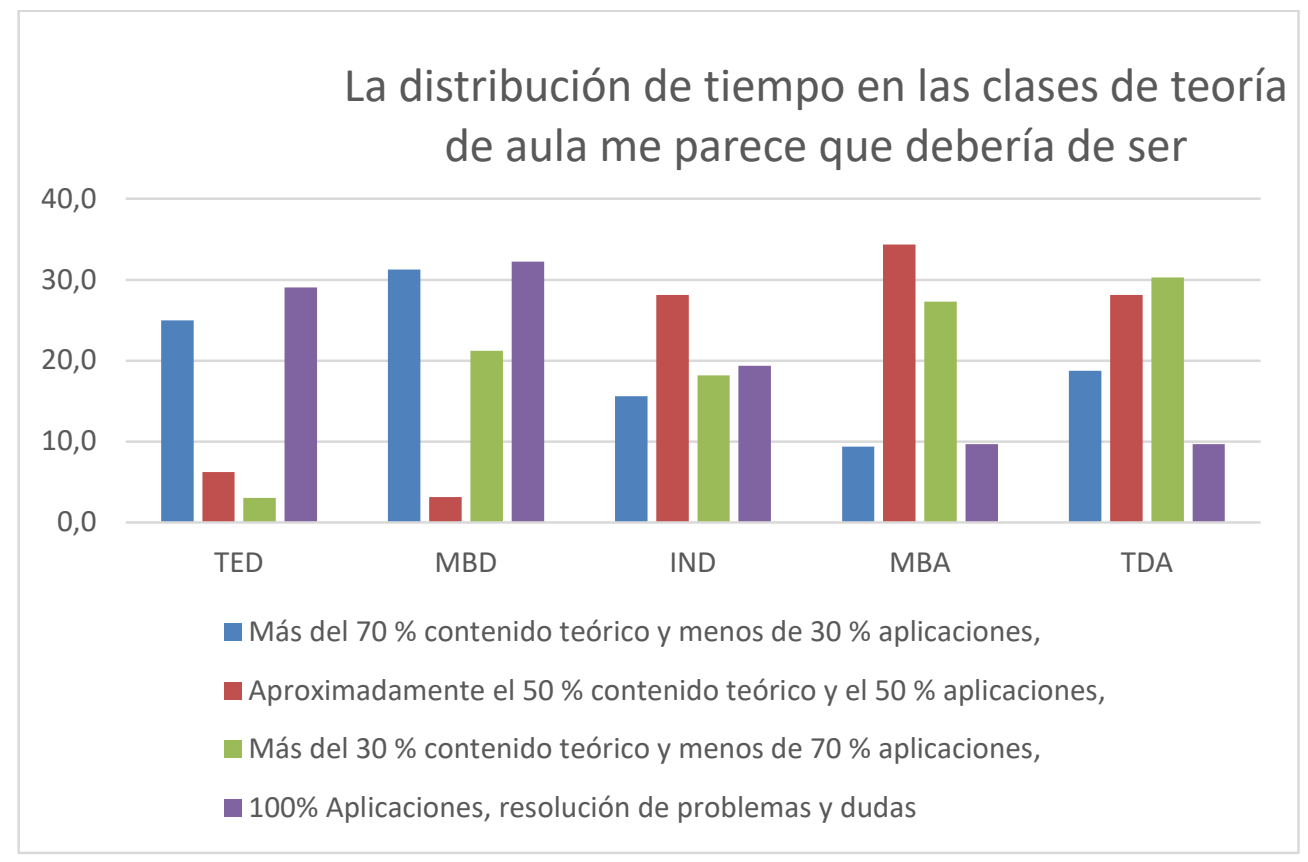

Fig. 7. P2: La distribución de tiempo en las clases de teoría de aula me parece que debería de ser...

La Fig. 7 muestra las preferencias de los estudiantes en relación a los contenidos teóricos y prácticos. Vale la pena destacar que en esta asignatura, con la metodología de DI, se dedica mayor porcentaje del tiempo de las clases a la discusión de aplicaciones y resolución de problemas que en la metodología tradicional (aproximadamente 50/50). Los alumnos se muestran, en general, partidarios de una distribución homogénea entre el tratamiento teórico y práctico de la asignatura (50/50), o en menor medida a los contenidos teóricos (30//70. El resto de opciones son minoritarias. 


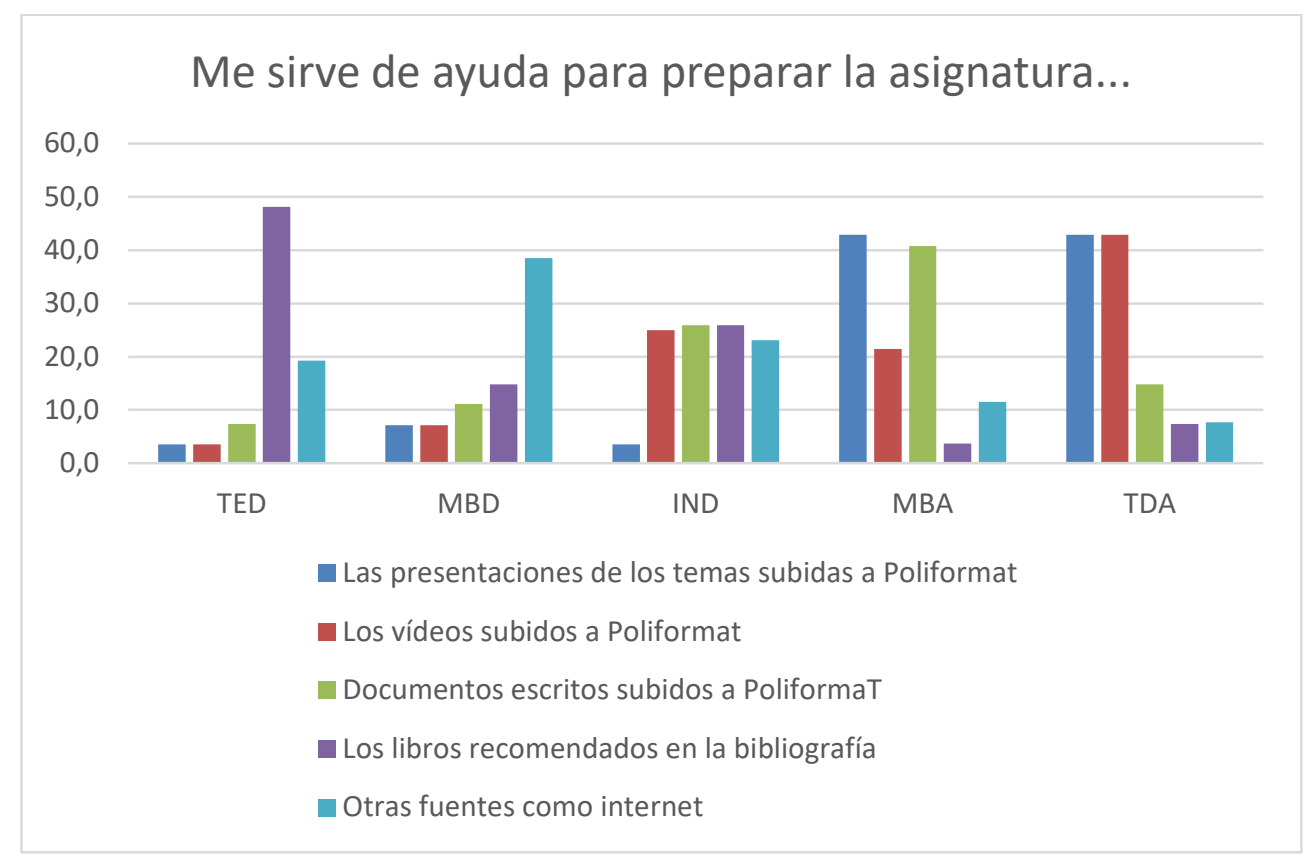

Fig. 8. P3: Me sirve de ayuda para preparar la asignatura ...

En cuanto a las preferencias sobre los recursos a utilizar (Fig. 8), los más valorados son aquellos de aplicación directa en la marcha de la asignatura (presentaciones/vídeos), frente a aquellos otros más generales. Destaca la poca valoración de los libros recomendados en la bibliografía. Aunque sería deseable que los alumnos consultaran habitualmente los recursos bibliográficos, hay que tener en cuenta que esta asignatura no dispone de textos que engloben la asignatura, ni de libro de referencia.

La capacidad de trabajar en equipo es una competencia muy demandada en la sociedad actual, y esencial en el desarrollo de la DI. A partir de los resultados de la encuesta podemos apreciar 


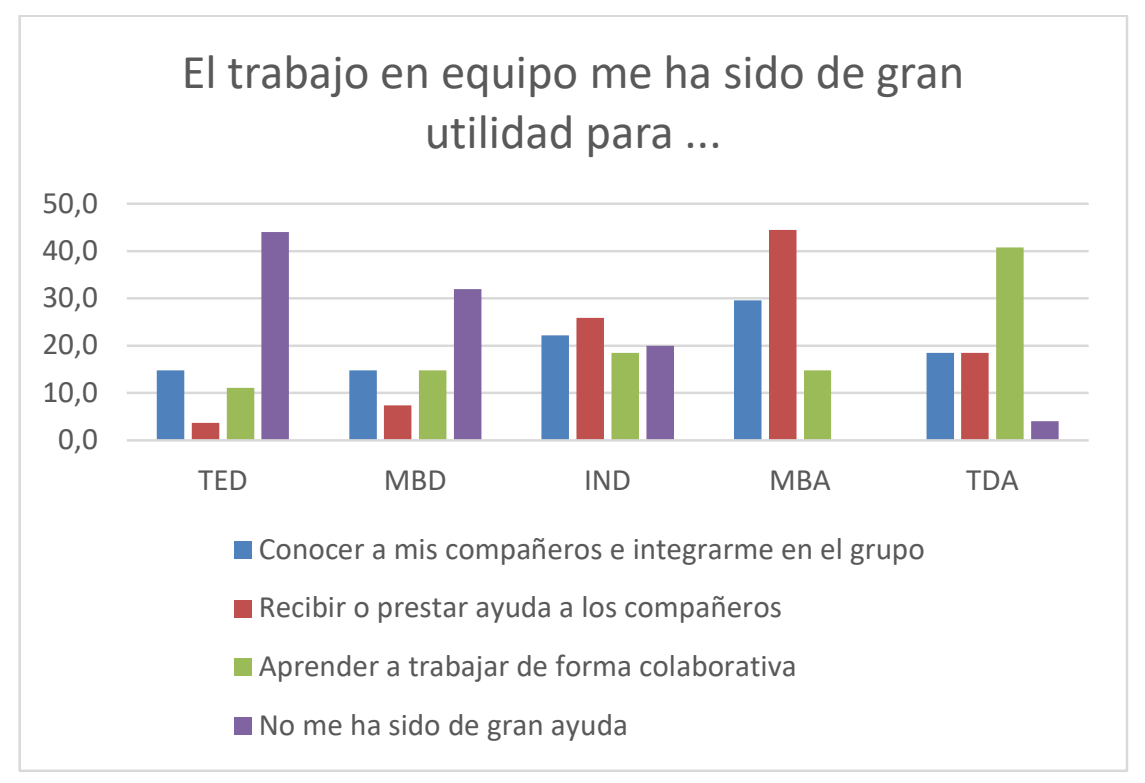

Fig. 9. P4: El trabajo en equipo, me ha sido de gran utilidad para...

como los alumnos son conscientes de su importancia, poniendo en valor el aprendizaje colaborativo (véase Fig. 9).

Por último, planteamos una pregunta de respuesta abierta, acerca de las posibles mejoras a introducir en la metodología seguida. La opción más demandada (Fig. 10) es la de realizar más problemas en clase. Lo mejor valorado son los vídeos, los exámenes de autoevaluación para resolver en casa y, en general, el desarrollo de las clases. Es decir, los alumnos refrendan el diseño y organización de la asignatura, valorando positivamente el papel de las herramientas que se ha puesto a su disposición.

\section{En qué se podría mejorar}

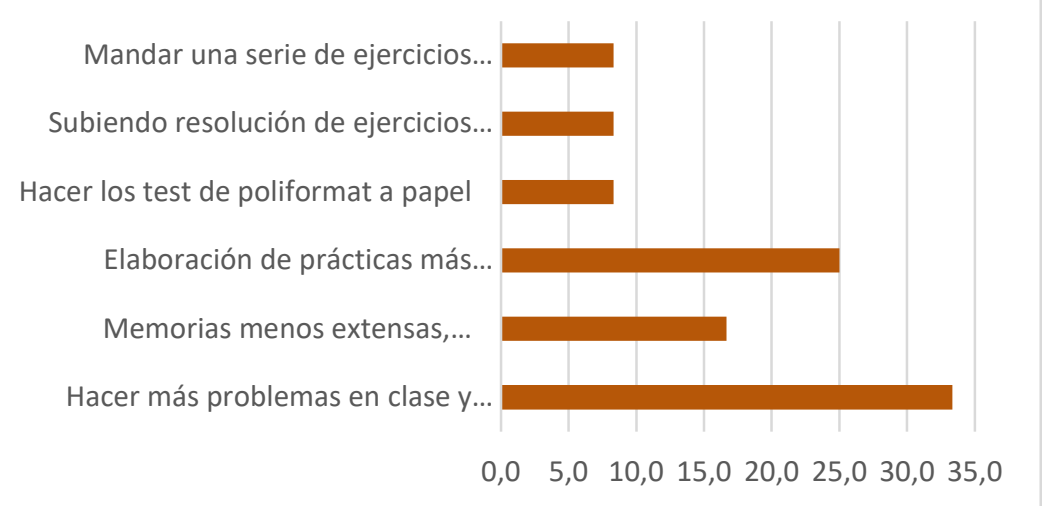

Fig.10. P5: Se podría mejorar...

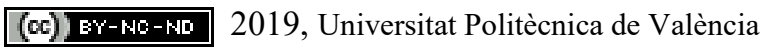




\section{Conclusiones}

El análisis de los resultados pone de manifiesto que los alumnos trabajan de forma continua. Las visitas están distribuidas en los 7 días de la semana y con bastante antelación a la fecha del examen. Esto está relacionado con que son alumnos muy trabajadores y con alta motivación por la asignatura. Tienen capacidad de planificar bien el tiempo, lo que se aprecia en los patrones de acceso a la plataforma.

En general, puede apreciarse que los vídeos se consultan preferentemente cuando se presenta el tema y en menor medida en las fechas previas al examen. El material disponible, organizado mediante la herramienta Lessons, recibe una buena valoración por parte de los alumnos (en especial los vídeos).

En concordancia con el diseño propuesto para la asignatura, sus preferencias respecto a la distribución de tiempo en las clases (teoría/aplicaciones) son entre 30 y 50 de teoría, y entre 70 y 50 de aplicación.

En resumen, la herramienta Lessons permite organizar de forma satisfactoria la docencia inversa y su utilización es bien valorada por los estudiantes.

\section{Agradecimientos}

Los autores agradecen al Instituto de Ciencias de la Educación de la Universitat Politècnica de València su apoyo al grupo de Innovación e-MACAFI y al Proyecto PIME/2018/B25.

\section{Referencias}

Ardid, M., Gómez-Tejedor, J. A., Meseguer-Dueñas, J. M., Riera, J., y Vidaurre, A. (2015). "Online exams for blended assessment. Study of different application methodologies". Computers \& Education, vol. 81, p. 296-303.

Argente, E., García-Fornes, A. y EspinosA, A. (2016). “Aplicando la metodología Flipped-Teaching en el Grado de Ingeniería Informática: una experiencia práctica”. En XXII Jornadas sobre la Enseñanza Universitaria de la Informática (JENUI 2016) (6-8 de julio 2016. Almería). Actas de las XXII Jenui. Universidad de Almería. 221-228.

Bonwell, C. C., y Eison, J. A. (2005). “Active learning: creating excitement in the classroom”. San Francisco: Jossey-Bass a Wiley brand.

Chen, Y., WAng, Y., Kinshuk, y Chen, N. S. (2014). "Is FLIP enough? or should we use the FLIPPED model instead?" Computers and Education, vol. 79, p. 16-27.

GARrison, D. R., y VAUghan, N. D. (2013). "Institutional change and leadership associated with blended learning innovation: Two case studies". Internet and Higher Education, vol.18, p. 24-28. 
Gómez-Tejedor, J.A., Vidaurre, A., Tort-Ausina, I., Molina Mateo, J., Serrano, M.-A., Meseguer-Dueñas, J.M., MArtínez SAla, R.M., Quiles, S. y RierA, J. (2019). "Effectiveness of flip teaching on engineering students' performance in the physics lab". Enviado para su publicación a Computers \& Education.

Jovanovic, J., Mirriahi, N., GaŠević, D., Dawson, S., And Pardo, A., (2019), "Predictive Power of Regularity of Pre-Class Activities in a Flipped Classroom," Computers \& Education, vol 134, p. 156168.

Lage, M. J., Platt, G. J., y Treglia, M. (2000). "Inverting the classroom: A gateway to creating an inclusive learning environment". Journal of Economic Education, vol. 31, issue 1, p. 30-43.

Lemley, E.C., Jassemnejad, B., Judd, E., Ring, B.P., Henderson, A.W. y Armstrong G.M. (2013). "Implementing a Flipped Classroom in Thermodynamics". En Transactions of the American Society for Engineering Education (ASEE Annual Conference \& Exposition, 23-26 junio 2013. Atlanta). Paper ID \#7815, Paginas : 23.703.1- 23.703.8.

Meyers, C., y Jones, T. B. (1993). "Promoting active learning : strategies for the college classroom". Jossey-Bass.

SAKAI. Sakai. Learning Management System. < https://sakaiproject.org/> [Consulta: 13 de marzo de 2019].

Sun, Z., XiE, K., \& ANDERMAn, L. H. (2018). “The role of self-regulated learning in students' success in flipped undergraduate math courses". Internet and Higher Education, vol. 36, p. 41-53.

Terrasa Barrena, S. y Andreu GarcíA, G. (2015). "Cambio a metodología de clase inversa en una asignatura obligatoria". En Actas del simposio-taller previo Jornadas sobre la Enseñanza Universitaria de la Informática (JENUI 2015) sobre Estrategias y herramientas para el aprendizaje y la evaluación (8-10 de julio 2015. Andorra). Actas de las XXI Jenui. La Salle Open University de Andorra. 32-37.

Tomas, L., Evans, N. (Snowy), Doyle, T., And Skamp, K., (2019). “Are First Year Students Ready for a Flipped Classroom? A Case for a Flipped Learning Continuum," International Journal of Educational Technology in Higher Education, vol. 16, p 1-22.

Universitat Politècnica DE VAlÈnCIA. Docencia inversa /Flip teaching. < http://www.upv.es/contenidos/SMAT/etsa/1041403normalc.html > [Consulta: 13 de marzo de 2019]

Universitat Politècnica DE VALÈnCIA. Clase inversa o 'flipped classroom'. < http://www.upv.es/noticias-upv/noticia-6615-clase-inversa-o-es.html> [Consulta: 13 de marzo de 2019].

Universitat Politècnica DE VAlÈncia. Proyecto Docencia Inversa UPV. < https://docenciainversa.blogs.upv.es/el-proyecto/proyecto-clase-inversa-upv/> [Consulta: 13 de marzo de 2019].

Universitat Politècnica De ValÈncia. PoliformaT. < https://poliformat.upv.es/portal/> [Consulta: 13 de marzo de 2019].

VALVERdE RAmos, A (2014). "Una experiencia de enseñanza inversa en un curso de matemáticas en Ingeniería Informática“. En En XX Jornadas sobre la Enseñanza Universitaria de la Informática (JENUI 2014) (9-11 de julio 2014. Oviedo). Actas de las XX Jenui. Universidad de Oviedo. 435-442.

Vidaurre, A., Meseguer-Dueñas, J.M., Riera, J, Molina Mateo, J., Molina Mateo, J., GómezTejedor, J.A., Serrano, M.-A., Gamiz-GonzÁleA, M. A.;. y. TorT-AusinA, I., (2018). "Is the Lessons tool useful to support students learning?. International Conference on Innovation,Documentation and Education (Valencia, 14th-16th November INNODOCT/18. 119-128 
Vo H. M., Zhu C., AND DieP N. A., (2017). "The Effect of Blended Learning on Student Performance at Course-Level in Higher Education: A Meta-Analysis," Studies in Educational Evaluation., vol. 53, p. $17-28$. 